\title{
Sterile neutrino searches at tagged kaon beams
}

\author{
Luis A. Delgadillo* and Patrick Huber ${ }^{\dagger}$ \\ Center for Neutrino Physics, Department of Physics, Virginia Tech, Blacksburg, Virginia 24061, USA
}

(Received 6 November 2020; accepted 23 January 2021; published 19 February 2021)

\begin{abstract}
Tagged kaon beams are attractive neutrino sources, which would provide flavor pure $\nu_{e}$ beams with exactly measured normalization. We point out that this also leads to an antitagged flavor pure $\nu_{\mu}$ beam, with equally well-known normalization. Exposing a $1 \mathrm{kt}$ liquid argon detector at a baseline of $1 \mathrm{~km}$ to this combination of unique beams allows us to decisively test recent indications by IceCube and Neutrino-4 of sterile neutrino oscillations in the multi-eV range.
\end{abstract}

DOI: $10.1103 /$ PhysRevD.103.035018

\section{INTRODUCTION}

The decisive measurement of a nonzero reactor mixing angle [1-3] has marked the beginning of an era of precision neutrino mixing measurements. The current neutrino oscillation data have determined the neutrino mass squared differences, $\Delta m_{21}^{2}$ and $\left|\Delta m_{31}^{2}\right|$, and the mixing angles $\sin ^{2}\left(\theta_{i j}\right)$ (with $i j=\{12,13,23\}$ ) of the lepton mixing matrix [4]. Nevertheless, there exist persistent hints from different experiments, notably LSND [5] and MiniBooNE [6], as well as reactor experiments [7-11] that may indicate the existence of a fourth neutrino species, a sterile neutrino $\nu_{s}$ with a mass of $\mathcal{O}(1) \mathrm{eV}$ mixing with active neutrinos. For a review on global sterile neutrino oscillations; see, e.g., Ref. [12] and references therein. While sterile neutrino oscillation would be a simple explanation, if applied to both the $\nu_{\mu}$-appearance results (LSND and MiniBooNE) and the reactor indications, it also would predict the disappearance of $\nu_{\mu}$, which has not been observed. On the contrary, strong limits have been placed on this mode over the years, and thus a sterile neutrino interpretation of all these anomalous results seems unlikely. For masses of the new state above an $\mathrm{eV}$, also cosmological bounds become an issue, and direct bounds from KATRIN apply as well [13]. More recently, two experiments provided indications of multi-eV scale oscillations. Specifically, the Neutrino-4 experiment reports a best-fit $\Delta m^{2} \simeq 7 \mathrm{eV}^{2}$ [14], and IceCube reports a best-fit value of $\Delta m^{2} \simeq 4.5 \mathrm{eV}^{2}[15,16]$. The multi-eV region is difficult to access for reactor neutrino experiments due to the smearing out of oscillations because of the reactor core

\footnotetext{
*luisd@vt.edu

pahuber@vt.edu
}

Published by the American Physical Society under the terms of the Creative Commons Attribution 4.0 International license. Further distribution of this work must maintain attribution to the author(s) and the published article's title, journal citation, and DOI. Funded by SCOAP ${ }^{3}$. size. It is not yet clear if these new indications are compatible with the other $\mathrm{eV}$-scale indications and if they will persist with increased statistics and/or a more careful assessment of systematic uncertainties. In this paper, we will investigate tagged kaon beams [17], to study multi-eV scale neutrino oscillation directly and with extremely wellconstrained systematics.

A recent example of a proposal to build a tagged kaon beam is the Enhanced Neutrino Beams from Kaon Tagging (ENUBET) beam line technology [18] based on the reconstruction of positrons from the three-body semileptonic $K^{+} \rightarrow e^{+} \nu_{e} \pi^{0}$ decay, aimed to determine the absolute $\nu_{e} / \nu_{\mu}$ flux at $1 \%$ level.

The basis for this type of tagged beam is a conventional narrow band beam with a short transfer line approximately $20 \mathrm{~m}$ followed by a $40 \mathrm{~m}$ long decay tunnel surrounded by positron detectors [19]. Primary protons of $120 \mathrm{GeV}$ in energy are impinged on a target to produce secondary hadrons $(\pi, K)$ which are captured, sign selected, and transported farther down to the instrumented decay tunnel with a momentum spread of $\pm 20 \%$ centered at $8.5 \mathrm{GeV}$ [18]. The positrons are identified in the decay tunnel by calorimetric techniques, and the beam line is optimized to enhance the $\nu_{e}$ component from the three-body semileptonic decay and suppress to a negligible level the $\nu_{e}$ contamination from muon decays [18]; the decay tunnel is too short for muon decays to produce a significant fraction of muon neutrinos. In ENUBET, the rate of positrons provides a direct measurement of the $\nu_{e}$ produced in the tunnel. Also, the three-body decay of the kaon produces a wider neutrino energy distribution than the twobody decay of the pion, $\pi^{+} \rightarrow \mu^{+}+\nu_{\mu}$, as can be seen in Fig. 1; thus, an energy cut can be used to effectively control any remaining backgrounds after tagging. Neutrinos from this type of source will oscillate as usual, since the precision of neither the time nor the energy measurement on the positron will allow us to determine the mass eigenstate produced, and thus all that tagging does is fix the beam normalization and baseline traveled. 
The Neutrino-4 indication is observed in the $\bar{\nu}_{e} \rightarrow \bar{\nu}_{e}$ channel, and the IceCube indication is observed in the $\nu_{\mu} \rightarrow$ $\nu_{\mu}$ channel only; thus, no specific prediction for an effect in $\nu_{\mu} \leftrightarrow \nu_{e}$ channels arises. A direct test of those indications is therefore best obtained by using the same channels as the original results. ${ }^{1}$ Therefore, we will focus the analysis here on these two disappearance channels.

\section{EXPERIMENTAL FRAMEWORK}

In this section, we describe the experimental setup and the assumptions that we use in the present analysis. The predicted event rates were calculated based on neutrino fluxes provided by the ENUBET Collaboration $[18,20]$. We consider a $1 \mathrm{kt}$ liquid argon detector with energy resolution which follows a Gaussian distribution with a width of $\sigma(E)=17 \% / \sqrt{E(\mathrm{GeV})}$ for electrons and $\sigma(E)=$ $10 \% / \sqrt{E(\mathrm{GeV})}$ for muons; a total of 50 bins in the energy interval of $0-10 \mathrm{GeV}$ were considered $[19,20]$. All calculations are performed with GLoBES [21,22] using the $\mathrm{N}$-flavor oscillation engine of Refs. [23,24].

The signal is obtained from the survival probability of electron neutrinos $\left(\nu_{e} \rightarrow \nu_{e}\right)$ stemming from the $K^{+}$in the beam that decay into $e^{+}+\nu_{e}$, which then interact in the liquid argon detector through the weak charged current. The yields of kaons transported to the entrance of the decay tunnel are $1.69 \times 10^{-3} K^{+} / \mathrm{PoT}$ (protons on target) for $120 \mathrm{GeV}$ protons. The tagged $\nu_{e}$ flux is assumed to be $99 \%$ pure. The largest source of beam related background in the detector is due to neutral current coherent $\pi^{0}$ production: the $\pi^{0}$ decays into two photons. For pion energies below approximately $1 \mathrm{GeV}$, the opening angle is large enough to cleanly reconstruct both photons, and thus no confusion with a $\nu_{e}$ charged-current event arises. At higher energies, however, the two photons are more collinear and may no longer be reconstructed as two particles; hence, these neutral currents events may be misidentified as chargedcurrent $\nu_{e}$ events. Liquid argon (LAr) detectors have very fine granularity and as a result very good particle identification. In particular, photon-induced showers can be recognized by the gap between the vertex and the start of the shower. Without going into the details of event reconstruction, we estimate the rate of coherent, neutral current $\pi^{0}$ production. The cross section for neutral current coherent $\pi^{0}$ production has been measured by MINOS on iron [25] and by NOvA on carbon [26]; we use a theoryderived scaling factor of $(A / 12)^{2 / 3}$ to translate these results for argon in accordance with the Berger-Sehgal model [27]. Expected $\pi^{0}$ rates were found to be of order $0.1 \%$ compared to our signal $\nu_{e}$ events and thus can be neglected for this analysis. The beam normalization is know at the $1 \%$ level due to the high kaon tagging efficiency [18].

\footnotetext{
${ }^{1}$ Note, that as long as CPT symmetry holds $\bar{\nu}_{\alpha} \rightarrow \bar{\nu}_{\alpha}$ has to have the same oscillation probability as $\nu_{\alpha} \rightarrow \nu_{\alpha}$.
}

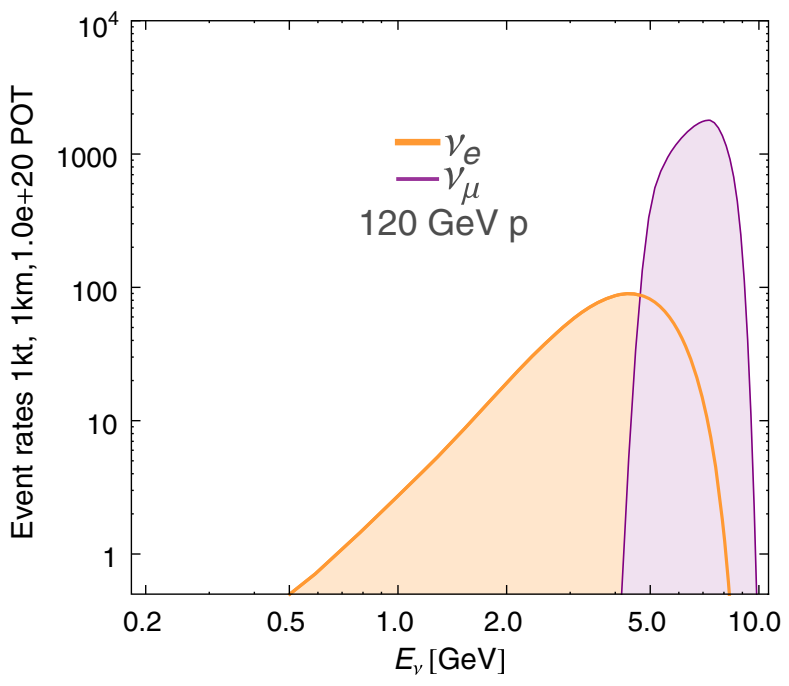

FIG. 1. Expected event rates assuming a $1 \mathrm{kt}$ liquid argon detector at baseline $L=1 \mathrm{~km}$ for $120 \mathrm{GeV}$ protons with a power beam of $10^{20} \mathrm{PoT} / \mathrm{yr}$.

Similarly, muon neutrinos can be selected at the neutrino detector using radius-energy correlations. We performed a $5 \mathrm{GeV}$ energy cut to avoid contributions from untagged $\pi^{+}$; since the branching ratio for the semileptonic decay $\mathrm{K}^{+} \rightarrow$ $e^{+} \nu_{e} \pi^{0}$ is well known, 5\%, and the number of kaon decays in this mode is fixed by tagging, we can use the equally well-known branching ratios for the muon neutrino generating decay modes $K^{+} \rightarrow \mu^{+} \nu_{\mu}(60 \%)$ and $K^{+} \rightarrow \mu^{+} \nu_{\mu} \pi^{0}$ (3\%) to know the muon neutrino flux with the same accuracy as the electron neutrino flux. Final states here are charged-current $\nu_{\mu}$ interactions with the detector. As in the electron neutrino case, neutral current background events were found to be of order $0.1 \%$ compared to signal and thus negligible.

Placing a $1 \mathrm{kt}$ liquid argon detector at a distance of $1 \mathrm{~km}$ from the decay pipe, we obtain $1568 \nu_{e}$ events and 24,603 $\nu_{\mu}$ events $/ y{ }^{2}{ }^{2}$ Detection efficiencies in this type of detector are close to $100 \%$, and for simplicity, we neglect them; a simple increase of running time or detector mass by $10 \%-$ $20 \%$ will be required to compensate for this approximation. For a few-GeV beam, a distance of $L=1 \mathrm{~km}$ yields $L / E_{\nu} \simeq 0.2(\mathrm{~km} / \mathrm{GeV})$, which corresponds to an oscillation with $\Delta m^{2} \simeq 5 \mathrm{eV}^{2}$. The $\nu_{\mu}$ energy $E_{\nu}$ spectrum peaks at approximately $7 \mathrm{GeV}$, while the $\nu_{e}$ energy spectrum peaks around $4 \mathrm{GeV}$ as shown in Fig. 1. We do not include the appearance channels in our analysis and have confirmed that their inclusion would not impact our results in an appreciable manner.

\footnotetext{
${ }^{2}$ This includes cuts on the beam radius with acceptances of $24 \%$ for $\nu_{e}$ and $34 \%$ for $\nu_{\mu}$ [28].
} 


\section{STERILE NEUTRINO SEARCHES}

Our treatment of the $3+1$ framework of neutrino oscillations follows the leptonic mixing matrix parametrization [12]

$U=R\left(\theta_{34}\right) \tilde{U}\left(\theta_{24}, \delta_{24}\right) R\left(\theta_{14}\right) R\left(\theta_{23}\right) \tilde{U}\left(\theta_{13}, \delta\right) \tilde{U}\left(\theta_{12}, \delta_{12}\right)$,

where $R\left(\theta_{i, j}\right)$ are orthogonal $4 \times 4$ matrices on the $(i, j)$ plane, $\tilde{U}\left(\theta_{i, j}, \delta_{i, j}\right)$ are $4 \times 4$ unitary matrices on the $(i, j)$ plane, and $\delta$ is the standard Dirac $C P$-violating phase; under the short baseline approximation, all extra phases are zero (i.e., there will be no additional $C P$ violation in the $3+1$ scenario). The probability for a neutrino produced in the flavor eigenstate $\nu_{\alpha}$ to be observed as flavor $\nu_{\beta}$ after traveling some distance $L$ in vacuum and having energy $E$ is

$$
\begin{aligned}
P_{\alpha \beta}= & \delta_{\alpha \beta}-4 \sum_{i>j} \mathcal{R}\left[U_{\alpha i}^{*} U_{\alpha j} U_{\beta i} U_{\beta j}^{*}\right] \sin ^{2}\left(1.27 \Delta m_{i j}^{2} \frac{L}{E}\right) \\
& +2 \sum_{i>j} \mathcal{I}\left[U_{\alpha i}^{*} U_{\alpha j} U_{\beta i} U_{\beta j}^{*}\right] \sin \left(2.54 \Delta m_{i j}^{2} \frac{L}{E}\right),
\end{aligned}
$$

where $U_{\alpha i}(\alpha=e, \mu, \tau, s ; i=1,2,3,4)$ are the elements of the leptonic mixing matrix, $E$ is the neutrino energy, $L$ is the beam to detector distance, and $\Delta m_{i j}^{2}=m_{i}^{2}-m_{j}^{2}$ are the squared mass splittings between the standard neutrino mass eigenstates $\nu_{1}, \nu_{2}, \nu_{3}$ and a $\nu_{4}$ sterile state.

Based on our simulated charged-current event rates and assuming a $10^{20} \mathrm{PoT} / \mathrm{yr}$ beam power on a $1 \mathrm{kt} \mathrm{LAr}$ detector, we obtain sensitivities for the hypothesis of sterile neutrino oscillation under a $(3+1)$ scenario assuming five years of beam operation. Interpretation of the ENUBET experimental data in terms of sterile neutrino oscillations allows us to test large values of $\Delta m_{41}^{2}$ and relatively sizable mixing between $\nu_{e}$ and $\nu_{s}$ states. This corresponds well with the parameter space regions indicated by the gallium results [29], Neutrino-4 results [14], and IceCube results $[15,16]$. Sensitivity contours were calculated based on the $\Delta \chi^{2}$ value for each parameter pair $\left(\Delta m_{41}^{2}, \sin ^{2} 2 \theta_{14}\right)$ and by determining the boundary of the corresponding

TABLE I. Relevant oscillation parameters in the $3+1$ scenario used in this analysis.

\begin{tabular}{lccc}
\hline \hline Standard PMNS & Value [NO] & Sterile parameter & Value \\
\hline$\theta_{12}$ & $33.2^{\circ}$ & $\delta_{24}$ & 0 \\
$\theta_{23}$ & $45^{\circ}$ & $\delta_{12}$ & 0 \\
$\theta_{13}$ & $9^{\circ}$ & $\theta_{34}$ & 0 \\
$\Delta m_{21}^{2}\left(10^{-5} \mathrm{eV}^{2}\right)$ & 7.5 & $\theta_{24}$ & Free \\
$\left|\Delta m_{31}^{2}\right|\left(10^{-3} \mathrm{eV}^{2}\right)$ & 2.6 & $\theta_{14}$ & Free \\
$\delta$ & 0 & $\Delta m_{41}^{2}\left(10^{-1}-10^{2} \mathrm{eV}^{2}\right)$ & Free \\
\hline \hline
\end{tabular}

exclusion/allowed regions by translating the $\Delta \chi^{2}$ to confidence levels using a $\chi^{2}$ distribution with 2 degrees of freedom assuming normal ordering (NO). For a recent discussion about the limitations of Wilks's theorem in disappearance searches with free beam normalization, see Ref. [30], which is not quite the same case as that considered here but gives an indication of the size of the resulting corrections. Based on Table I we consider different null hypotheses $H_{0}$ :

(i) $H_{0}$ : no oscillation. We compute data assuming no disappearance and fit the resulting Asimov dataset with finite value of $\Delta m^{2}$ and $\sin ^{2} 2 \theta$. The result is a sensitivity limit, shown as dashed lines.

(ii) $H_{0}$ : oscillation according the reactor antineutrino anomaly (RAA) best fit. We compute data assuming the best fit of the RAA is true and fit the resulting Asimov dataset with different value of $\Delta m^{2}$ and $\sin ^{2} 2 \theta$. The result are allowed regions (with closed contours), shown as solid lines.

(iii) $H_{0}$ : oscillation according the Neutrino-4 best fit. We compute data assuming the best fit of Neutrino- 4 is true and fit the resulting Asimov dataset with different value of $\Delta m^{2}$ and $\sin ^{2} 2 \theta$. The result are allowed regions (with closed contours), shown as solid lines.

(iv) $H_{0}$ : oscillation according the IceCube best fit. We compute data assuming the best fit of IceCube is true and fit the resulting Asimov dataset with different value of $\Delta m^{2}$ and $\sin ^{2} 2 \theta$. The result are allowed regions (with closed contours), shown as solid lines.

For all cases, we consider a combined fit of muon and electron neutrino disappearance and profile over the notshown $\theta_{i 4}$ mixing angles.

For this experimental setup, the normalization error of the signal becomes important, while the beam flux is very well known due to tagging the signal charged-current cross sections are subject to large uncertainties. We assume that the main physics program of ENUBET, which is cross section measurements, has reduced the resulting effective signal uncertainty to either $1 \%, 2 \%$, or $5 \%$, where we take $2 \%$ as default unless stated otherwise.

The effective two-flavor limit in the electron disappearance channel yields this simple oscillation probability,

$P_{e e}=1-4\left|U_{e 4}\right|^{2}\left(1-\left|U_{e 4}\right|^{2}\right) \sin ^{2}\left(1.27 \Delta m_{41}^{2} \frac{L}{E}\right)$,

and according to the parametrization Eq. (1), this case reduces to the effective two flavor oscillation

$$
P_{e e}=1-\sin ^{2} 2 \theta_{e e} \sin ^{2}\left(1.27 \Delta m_{41}^{2} \frac{L}{E}\right),
$$

where $\sin ^{2} 2 \theta_{e e}=4\left|U_{e 4}\right|^{2}\left(1-\left|U_{e 4}\right|^{2}\right)$ and $\theta_{e e}=\theta_{14}$ is the angle that encodes mixing. 


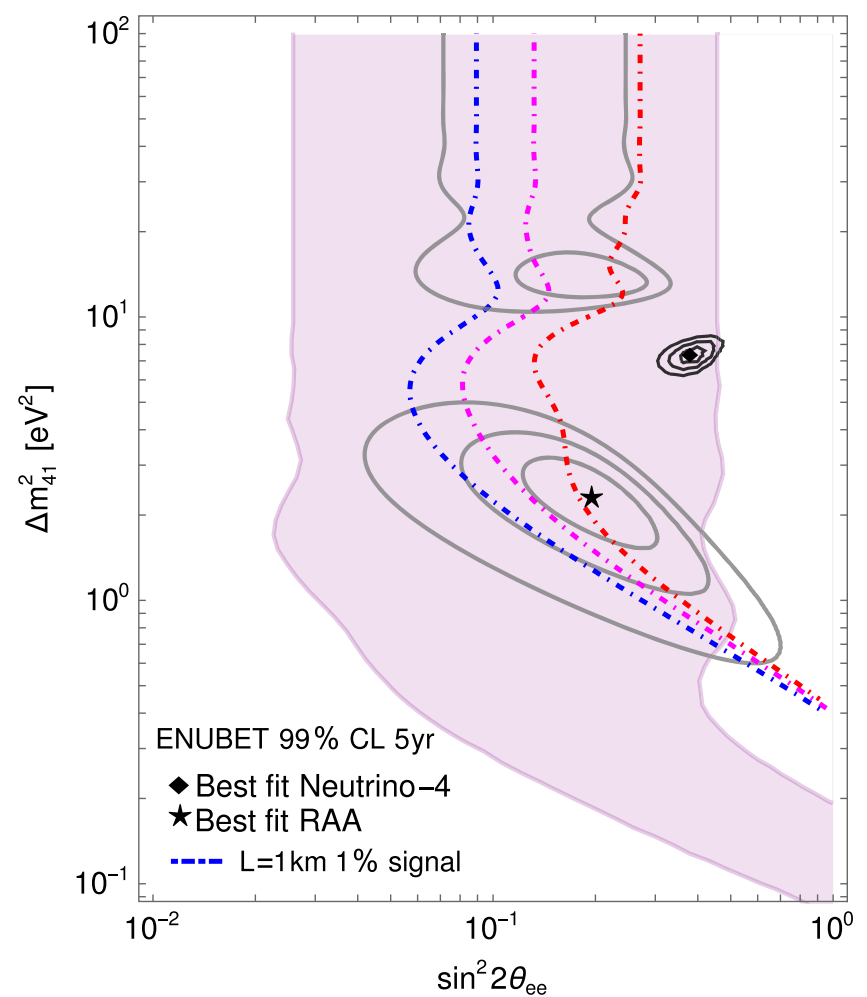

FIG. 2. Comparison of the expected sensitivities in the $\sin ^{2} 2 \theta_{e e}-\Delta m_{41}^{2}$ plane. The blue, magenta, and red dot-dashed lines correspond to $1 \%, 2 \%$, and $5 \%$ signal systematics at a baseline length of $L=1 \mathrm{~km}$. The black diamond point represents the best-fit point from Neutrino-4 [31], and the star represents the best fit of the reactor antineutrino anomaly RAA [7]. In addition, we show the $90 \%$ C.L. allowed region (purple shaded area) of the gallium anomaly JUN45 [29].

Figure 2 shows the sterile neutrino oscillation sensitivity at ENUBET in the $\sin ^{2} 2 \theta_{e e}-\Delta m_{41}^{2}$ plane at $99 \%$ C.L. for an exposure of $1 \mathrm{kt}$ assuming five years of beam operation. The blue, magenta, and red dot-dashed lines account for $1 \%, 2 \%$, and $5 \%$ signal normalization systematic. Also shown are the $1 \sigma, 2 \sigma$, and $3 \sigma$ preferred regions for the bestfit RAA [7] and Neutrino-4 [31] assuming 2\% signal normalization systematics.

Until recent results from IceCube $[15,16]$, no indication for sterile neutrino oscillation in the muon disappearance channel had been found. In the $3+1$ scenario with short baseline approximation, the muon neutrino disappearance probability is given by

$$
\begin{aligned}
P_{\mu \mu} & =1-4\left|U_{\mu 4}\right|^{2}\left(1-\left|U_{\mu 4}\right|^{2}\right) \sin ^{2}\left(1.27 \Delta m_{41}^{2} \frac{L}{E}\right) \\
& =1-\sin ^{2} 2 \theta_{\mu \mu} \sin ^{2}\left(1.27 \Delta m_{41}^{2} \frac{L}{E}\right),
\end{aligned}
$$

where $\left|U_{\mu 4}\right|=\cos \theta_{14} \sin \theta_{24}$ and the effective mixing angle $\theta_{\mu \mu}$ depends on both $\theta_{14}$ and $\theta_{24}$,

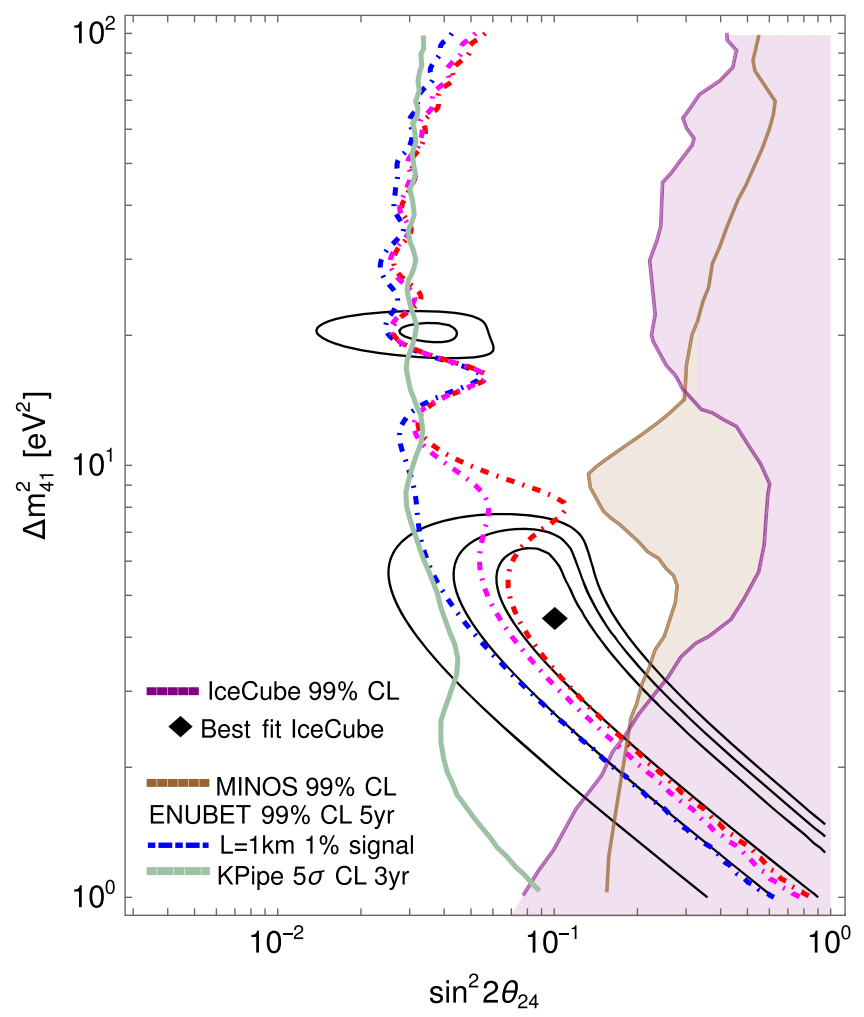

FIG. 3. Comparison of the expected sensitivities in the $\sin ^{2} 2 \theta_{24}-\Delta m_{41}^{2}$ plane $\left(\sin ^{2} 2 \theta_{\mu \mu} \approx \sin ^{2} 2 \theta_{24}\right)$. The blue, magenta, and red dot-dashed lines corresponds to a $1 \%, 2 \%$, and $5 \%$ signal systematics at baseline length of $L=1 \mathrm{~km}$. The black diamond point represents the best-fit point $90 \%$ C.L. from IceCube $[15,16]$, and shaded purple and brown areas are excluded by IceCube and MINOS [33], respectively.

$$
\sin ^{2} 2 \theta_{\mu \mu}=4 \cos ^{2} \theta_{14} \sin ^{2} \theta_{24}\left(1-\cos ^{2} \theta_{14} \sin ^{2} \theta_{24}\right) .
$$

Figure 3 shows the sterile neutrino oscillation sensitivity at ENUBET in the $\sin ^{2} 2 \theta_{24}-\Delta m_{41}^{2}$ plane at $99 \%$ C.L. for an exposure of $1 \mathrm{kt}$ assuming five years of beam operation. The blue, magenta, and red dot-dashed lines account for $1 \%, 2 \%$, and $5 \%$ signal normalization systematic. Also shown the $1 \sigma, 2 \sigma$, and $3 \sigma$ preferred regions assuming $2 \%$ signal normalization systematic for true $\sin ^{2} 2 \theta_{24}=0.1$ and $\Delta m_{41}^{2}=4.5 \mathrm{eV}^{2}$ from IceCube $[15,16]$. For comparison, we also show the sensitivity of another kaon decay based proposal, called Kpipe [32]; Kpipe is a proposed experiment to investigate sterile neutrinos from kaon decay at rest and is aimed to set the strongest limits in the muon neutrino disappearance channel. The expected number of $\nu_{\mu}$ events is $1.02 \times 10^{5}$ events/year in a 684 ton liquid scintillator detector, which comes in the shape of $120 \mathrm{~m}$ long pipe. Kpipe would have about an order of magnitude more signal events than the proposal we consider here and thus in principle has the potential to provide excellent sensitivity in this channel. 


\section{CONCLUSIONS}

In this paper, we test different hypotheses regarding simulated experimental data of the ENUBET beam line technology, we demonstrate the capabilities of tagged kaon beams in the electron and neutrino disappearance channel to investigate intriguing indications from the Neutrino-4 and IceCube collaborations. The strength of the setup considered is the vanishingly small systematic errors from the beam flux and a virtually background-free measurement. The drawback is the relatively low beam luminosity, as result of the need to tag kaon decays individually. The proposed setup is envisioned as add-on measurement to the cross section program of ENUBET. The physics case arises mainly from the Neutrino-4 result, which is in a $\Delta m^{2}$ region, which ultimately may be hard for reactor neutrino experiments to test decisively. The proposed setup could decisively test either indication, IceCube at the $5 \sigma$ level and Neutrino- 4 at the $10 \sigma$ level. This setup is not unique in this capability, and of course, dedicated facilities like nuSTORM [34] would provide superior sensitivity. In the hunt for the sterile neutrino, opportunistic measurements always have played a major role, and we point out that if ENUBET is built and the Neutrino-4 indication persists, the setup in this paper would present such an opportunity.

\section{ACKNOWLEDGMENTS}

We acknowledge useful discussions with R. Pestes and J. M. Berryman. This work was supported by the U.S. Department of Energy Office of Science under Award No. DE-SC00018327.
[1] F. An et al. (Daya Bay Collaboration), Phys. Rev. Lett. 108, 171803 (2012).

[2] J. Ahn et al. (RENO Collaboration), Phys. Rev. Lett. 108, 191802 (2012).

[3] Y. Abe et al. (Double Chooz Collaboration), J. High Energy Phys. 10 (2014) 086; 02 (2015) 074(E).

[4] P. Zyla et al. (Particle Data Group), Prog. Theor. Exp. Phys. (2020), 083C01.

[5] A. Aguilar-Arevalo et al. (LSND Collaboration), Phys. Rev. D 64, 112007 (2001).

[6] A. Aguilar-Arevalo et al. (MiniBooNE Collaboration), Phys. Rev. Lett. 121, 221801 (2018).

[7] G. Mention, M. Fechner, Th. Lasserre, Th. A. Mueller, D. Lhuillier, M. Cribier, and A. Letourneau, Phys. Rev. D 83, 073006 (2011).

[8] Y.-J. Ko et al. (NEOS Collaboration), Phys. Rev. Lett. 118, 121802 (2017).

[9] H. Almazán et al. (STEREO Collaboration), Phys. Rev. Lett. 121, 161801 (2018).

[10] I. Alekseev et al. (DANSS Collaboration), Phys. Lett. B 787, 56 (2018).

[11] J. M. Berryman and P. Huber, arXiv:2005.01756.

[12] M. Dentler, A. Hernández-Cabezudo, J. Kopp, P. A. N. Machado, M. Maltoni, I. Martinez-Soler, and T. Schwetz, J. High Energy Phys. 08 (2018) 010.

[13] M. Aker et al. (KATRIN Collaboration), Phys. Rev. Lett. 123, 221802 (2019).

[14] A. Serebrov et al., arXiv:2005.05301.

[15] M. G. Aartsen et al. (IceCube Collaboration), Phys. Rev. Lett. 125, 141801 (2020).

[16] M. Aartsen et al. (IceCube Collaboration), Phys. Rev. D 102, 052009 (2020).
[17] R. H. Bernstein, F. Borcherding, D. Jovanovic, M. J. Lamm, and F. Vannucci, FERMILAB-PROPOSAL-0788 (1990).

[18] A. Longhin, L. Ludovici, and F. Terranova, Eur. Phys. J. C 75, 155 (2015)

[19] F. Acerbi et al., arXiv:1901.04768.

[20] F. Pupilli (private communication).

[21] P. Huber, M. Lindner, and W. Winter, Comput. Phys. Commun. 167, 195 (2005).

[22] P. Huber, J. Kopp, M. Lindner, M. Rolinec, and W. Winter, Comput. Phys. Commun. 177, 432 (2007).

[23] J. Kopp, Int. J. Mod. Phys. C 19, 523 (2008).

[24] J. Kopp, M. Lindner, T. Ota, and J. Sato, Phys. Rev. D 77, 013007 (2008).

[25] P. Adamson et al. (MINOS Collaboration), Phys. Rev. D 94, 072006 (2016).

[26] M. Acero et al. (NOvA Collaboration), Phys. Rev. D 102, 012004 (2020).

[27] C. Berger and L. M. Sehgal, Phys. Rev. D 79, 053003 (2009).

[28] A. Longhin, Novel neutrino beams, Neutrino 2020 Conference (2020).

[29] J. Kostensalo, J. Suhonen, C. Giunti, and P. C. Srivastava, Phys. Lett. B 795, 542 (2019).

[30] P. Coloma, P. Huber, and T. Schwetz, Eur. Phys. J. C 81, 2 (2021).

[31] A. Serebrov et al. (NEUTRINO-4 Collaboration), Pis'ma Zh. Eksp. Teor. Fiz. 109, 209 (2019).

[32] S. Axani, G. Collin, J. M. Conrad, M. H. Shaevitz, J. Spitz, and T. Wongjirad, Phys. Rev. D 92, 092010 (2015).

[33] P. Adamson et al. (Daya Bay and MINOS+ Collaborations), Phys. Rev. Lett. 125, 071801 (2020).

[34] D. Adey et al. (nuSTORM Collaboration), Phys. Rev. D 89, 071301 (2014). 